\title{
Spontaneous Symmetry-Breaking Vortex Lattice Transitions in Pure Niobium
}

\author{
M. Laver, ${ }^{1,2}$ E. M. Forgan, ${ }^{2}$ S. P. Brown, ${ }^{2}$ D. Charalambous,,${ }^{2,3}$ D. Fort, ${ }^{4}$ C. Bowell, ${ }^{2}$ S. Ramos, ${ }^{2}$ R. J. Lycett, ${ }^{2}$ \\ D. K. Christen, ${ }^{5}$ J. Kohlbrecher, ${ }^{6}$ C. D. Dewhurst, ${ }^{1}$ and R. Cubitt ${ }^{1}$ \\ ${ }^{1}$ Institut Laue-Langevin, BP 156, F-38042 Grenoble, France \\ ${ }^{2}$ School of Physics and Astronomy, The University of Birmingham, Birmingham B15 2TT, United Kingdom \\ ${ }^{3}$ Meteorological Service, Ministry of Agriculture, 28 Nikis Avenue, 1086 Lefkosia, Cyprus \\ ${ }^{4}$ Metallurgy and Materials Science, The University of Birmingham, Birmingham B15 2TT, United Kingdom \\ ${ }^{5}$ Solid State Division, Oak Ridge National Laboratory, Oak Ridge, Tennessee 37831-6393, USA \\ ${ }^{6}$ Paul Scherrer Institut, Villigen PSI, CH 5232, Switzerland
}

(Received 11 November 2005; published 25 April 2006)

\begin{abstract}
We report an extensive investigation of magnetic vortex lattice (VL) structures in single crystals of pure niobium with the magnetic field applied parallel to a fourfold symmetry axis, so as to induce frustration between the cubic crystal symmetry and hexagonal VL coordination expected in an isotropic situation. We observe new VL structures and phase transitions; all the VL phases observed (including those with an exactly square unit cell) spontaneously break some crystal symmetry. One phase even has the lowest possible symmetry of a two-dimensional Bravais lattice. This is quite unlike the situation in high- $T_{c}$ or borocarbide superconductors, where VL structures orient along particular directions of high crystal symmetry. The causes of this behavior are discussed.
\end{abstract}

DOI: 10.1103/PhysRevLett.96.167002

Niobium is one of the few type-II elemental superconductors and was used for the first experimental confirmation by small-angle neutron scattering (SANS) [1] of the existence of the Abrikosov vortex lattice [2]. Vortex lattice (VL) structures in $\mathrm{Nb}$ have since been extensively investigated [3-5]. However, following recent results exploring the effects of nonlocality and gap anisotropy on VL structure in tetragonal borocarbide and $d$-wave superconductors [6-9], we have reexamined the behavior in $\mathrm{Nb}$ with the magnetic field applied parallel to a fourfold [001] axis of the bcc crystal structure. We describe new VL structures at high fields and low temperatures and explore the transitions between phases. Surprisingly, all VLs break some crystal symmetry. Even when an exactly square VL unit cell is observed, it is not symmetrically oriented with respect to the underlying crystal lattice. We consider how the interactions between vortex lines could cause this spontaneous symmetry breaking and the distortions of the triangular VLs we observe.

The morphology of equilibrium vortex lattices in type-II superconductors reflects properties of the superconducting state such as anisotropy of the gap function and the Fermi surface. In the isotropic case, the Abrikosov VL [2] has a hexagonal coordination with an equilateral triangular halfunit cell [10]. Underlying anisotropies typically manifest themselves as equilibrium distortions from the equilateral triangle, or as transitions into other lattice structures. The crystal symmetry also strongly affects the VL coordination [11]; in pure $\mathrm{Nb}$, various structures have been observed depending on the applied field direction relative to the crystal lattice [3]. With its low Ginzburg-Landau parameter $\kappa \sim 0.77$, pure niobium displays an attractive interaction [12] between vortices; this becomes the usual repulsive interaction at intervortex spacings corresponding to
PACS numbers: 74.25.Qt, 61.12.Ex, 74.25.Dw, 74.70.Ad

an induction $B>B_{0} \sim 0.1 \mathrm{~T}[13,14]$. At fields near to $H_{c 1}$ applied to a sample with finite demagnetization factor, this leads to the formation of the intermediate mixed state (IMS), which contains regions of vortices of flux density $B_{0}$ and also Meissner regions. Here we reexamine the VL phases known to exist within the IMS and describe new symmetry-breaking VL structures at low temperatures and flux densities above $B_{0}$.

Our investigations using SANS were performed using the D22 instrument at the Institut Laue-Langevin in Grenoble and the SANS-I instrument at the Paul Scherrer Institut in Switzerland. In a typical high-resolution setup, cold neutrons with a mean wavelength of $13 \AA$ and a $10 \%$ FWHM wavelength spread were collimated over a distance of $18 \mathrm{~m}$ before reaching the sample. A $128 \times 128$ multidetector with $7.5 \mathrm{~mm}^{2}$ pixels at a distance of $18 \mathrm{~m}$ was used to detect the diffracted neutrons. The samples were two high-purity single crystals of niobium; the first (Nb-1) was a rod of length $14 \mathrm{~mm}$ and diameter $4 \mathrm{~mm}$ with its cylindrical axis coincident with the crystal [001] axis and had a residual resistance ratio $(R R R) \sim 1200$; the second $(\mathrm{Nb}-2)$ was a sphere of diameter $13 \mathrm{~mm}$ with $\mathrm{RRR} \sim 450$ and was used by Christen et al. in previous work [4]. Both samples had been annealed at temperatures in excess of $2300 \mathrm{~K}$ for at least $45 \mathrm{~h}$ in an ultimate vacuum better than $3 \times 10^{-10}$ Torr, and then heated briefly in an oxygen atmosphere at $\sim 700 \mathrm{~K}$; this reduces pinning of vortex lines at the sample surface [15]. The samples were mounted in a cryomagnet providing a magnetic field approximately parallel to the incident neutron beam. To acquire reciprocal space images of the VL, the cryomagnet and sample were rotated together by small angles about the vertical and horizontal axes, allowing us to collect integrated intensities while rocking the Bragg spots through the Ewald sphere. 
The VL was formed by applying the field above $T_{c}$ before cooling. Background data were collected at $T=10 \mathrm{~K}>$ $T_{c}$. The results from $\mathrm{Nb}-1$ and $\mathrm{Nb}-2$ were essentially identical, with minor variation in the transition fields between VL phases due to the slightly different sample purities.

Figure 1 shows typical VL diffraction patterns for field applied parallel to the [001] crystal axis. The fourfold symmetry of the [001] crystal direction in bcc $\mathrm{Nb}$ is reflected in these patterns, but does not necessarily imply fourfold symmetry in the structure of the VL. All of these patterns arise from the superposed effects of multiple VL domains existing in different regions of the sample. With the field exactly along [001], these domains would be degenerate and have equal populations. By rotating the [001] crystal axis away from the field direction, particular VL domains are favored, allowing us to confirm which diffraction spots belong to each domain.

Figure 1(a) shows the diffraction pattern in the temperature region around $4 \mathrm{~K}$ explored in previous work $[3,4]$. It
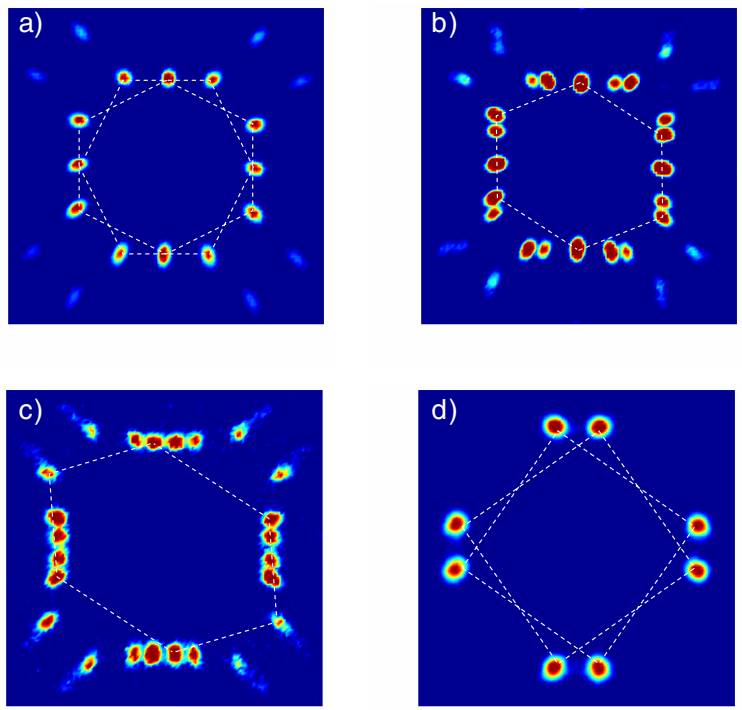

FIG. 1 (color online). Images of the diffraction pattern from the vortex lattice (VL) in $\mathrm{Nb}$ with the field applied parallel to the crystal [001] axis: at (a) $4 \mathrm{~K}$ and $150 \mathrm{mT}$ in sample $\mathrm{Nb}-1$, (b) $2 \mathrm{~K}$ and $200 \mathrm{mT}$ in $\mathrm{Nb}-1$, (c) $2 \mathrm{~K}$ and $325 \mathrm{mT}$ in Nb-2, and (d) $2 \mathrm{~K}$ and $350 \mathrm{mT}$ in $\mathrm{Nb}-2$. These pictures were prepared by summing several detector measurements (with backgrounds obtained at $T>T_{c}$ subtracted) over a range $\pm 1^{\circ}$ of rocking angles about the horizontal and vertical axes, allowing all Bragg reflections to be presented in a single image. Poisson noise from subtraction at the center of the picture has been masked. The crystal [010] and [100] directions are vertical and horizontal in these pictures. In (a) the dashed hexagons show the isosceles-triangular coordination of the two VL domains. In (b) and (c) the VL coordination is scalene triangular; one of the four VL domains present in this phase is indicated. In (d) the dashed squares show the two square VL domains present in a high-field, low-temperature phase. represents two VL domains, each having nearest neighbors arranged in an isosceles triangle. We determine VL reciprocal lattice vectors $q_{h k}$ by fitting 2D Gaussians to the Bragg spots, and confirm the $63.5^{\circ}$ interior isosceles angle. It is interesting to note that an isosceles triangle with height equal to base, that is, with angle $63.43^{\circ}$, can be obtained by applying pure shear to a square VL unit cell. We further observe, at an applied field $\mu_{0} H=200 \mathrm{mT}$, that the angles of the isosceles half-unit cell are preserved as the temperature is increased all the way to $T_{c 2}(H) \sim 5.4 \mathrm{~K}$. Clearly this cannot be described by standard GinzburgLandau theory, which would give a regular hexagon close to $T_{c} \sim 9.27 \mathrm{~K}$.

As the temperature is decreased, the isosceles triangle distorts into a scalene triangle, with all sides and angles unequal. The fourfold crystal symmetry then permits four equivalent VL domain orientations as illustrated in Fig. 2 and leads to complex diffraction patterns exemplified by Figs. 1(b) and 1(c). Each VL unit cell has the lowest possible symmetry of a two-dimensional Bravais lattice in spite of the fourfold crystal symmetry, which does not even determine how each VL domain orients. The structural transition from scalene to isosceles is continuous, as shown in Fig. 3.

To obtain the phase diagram as a function of VL flux density $B$ in the sample, we calculated the area of the real space unit cell from the reciprocal lattice vectors $q_{h k}$. In Fig. 4 we summarize the dependence of the unit cell shape on $B$ and $T$. At low $T$ the scalene VL converts into a square VL at low fields and also at high fields, as shown in Fig. 1(d). The high-field scalene and square phases have not been previously reported. We emphasize that although the high- and low-field square VL domains possess the rotational symmetry of the underlying crystal lattice, their orientations break the reflection symmetry of the crystal. This is very different from what is seen in high- $T_{c}$ and borocarbide superconductors [6-9].
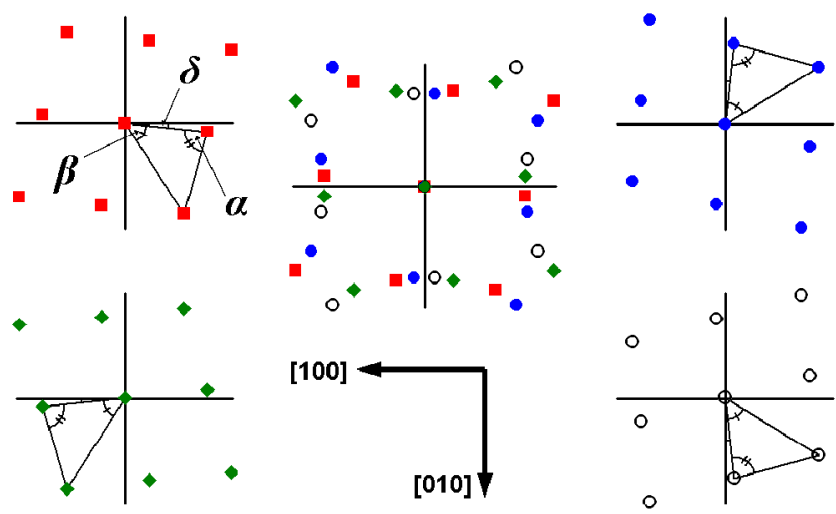

FIG. 2 (color online). Schematic representation in reciprocal space of the contribution of each of the four VL domains to the diffraction pattern observed for the scalene phase. The real space VL orientation can be visualized by rotating the indicated pattern by $90^{\circ}$ about the [001] axis. 


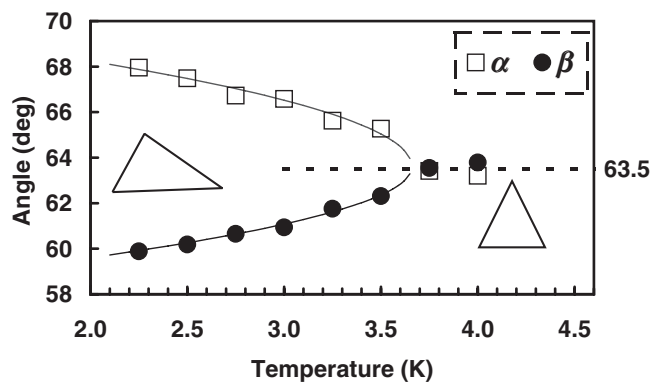

FIG. 3. The temperature dependence of vortex lattice morphology at $\mu_{0} H=200 \mathrm{mT}$ for sample Nb-1. The interior half-unit cell angles $\alpha, \beta$ are described in Fig. 2. The solid lines have been inserted as guides for the eye.

As expected, an essentially constant VL induction $B_{0}$ is observed in the IMS for a range of applied fields $\mu_{0} H \leq$ $155 \mathrm{mT}$. Within the IMS, the VL structure changes from square, through scalene, to isosceles triangular as the temperature is increased from 2 to $3 \mathrm{~K}$ [4]. For $B>B_{0}$ we find a large region of scalene phase. Close to $B_{0}$ we observe that square and scalene VL domains coexist in both samples with slightly different VL flux densities $\left(B_{\text {square }} \simeq B_{0}=\right.$ $100 \mathrm{mT}, B_{\text {scalene }} \geq 103 \mathrm{mT}$ at $2 \mathrm{~K}$ ), implying a first-order transition. The low-field square phase seems to be stable only within the IMS where the vortex lattice is at its equilibrium spacing (or perhaps under slight tension). Increasing the field to enter the mixed state places the VL under compression, which may be linked to the transition out of the square phase.

Figure 5(a) shows the change in shape of the VL with flux density at low temperatures. The first-order nature of the structural transition at low fields is clear. At high fields we find that the scalene VL continuously deforms into a different square lattice. Again, this high-field square VL

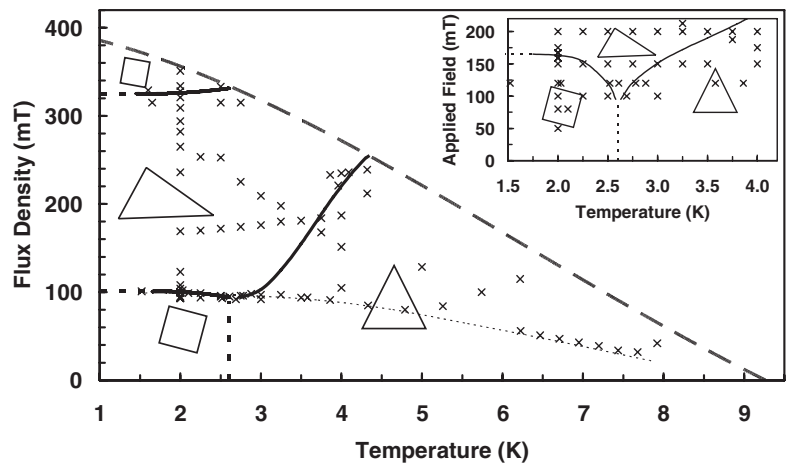

FIG. 4. Phase diagram, as a function of internal flux density $B$ and temperature $T$, of the various vortex lattice structures observed by SANS in sample $\mathrm{Nb}-1$. The dashed line shows $B_{c 2}(T)$, obtained from magnetization data; the crosses mark points at which diffraction patterns were observed; the thin dotted line shows $B_{0}(T)$ [14] and indicates the IMS region; the thick solid and dashed lines are guides to the eye indicating the boundaries between regions of different VL structures. The inset shows part of the phase diagram as a function of applied field $\mu_{0} H$ for this cylindrical sample. does not have an orientation determined by crystal symmetry. As seen in Fig. 5(b), the nearest-neighbor directions of this novel VL phase are $\pm 10.9(1)^{\circ}$ from the $\langle 100\rangle$ crystal axes. In the low-field square phase these angles are $\pm 14.7(1)^{\circ}$, in agreement with previous observations $[3,4]$. From Fig. 5(b) we see that one of the nearestneighbor directions in the scalene phase is within $1^{\circ}$ of a $\langle 100\rangle$ axis at low fields, even though there is no symmetry reason for exact alignment. As the field is increased, this direction smoothly changes [see Figs. 1(b)-1(d)] to that seen in the high-field square phase.

In high- $\kappa$ superconductors, the VL structure can be calculated by extending the London model to include a nonlocal relationship between supercurrent and vector potential arising from the finite size of Cooper pairs $[7,16]$. By this mechanism, coupling between the VL and crystal anisotropy of the Fermi velocity can account for many features of the VL transitions in $\mathrm{V}_{3} \mathrm{Si}$ [17], which has $\kappa \sim$ 30 and in the nickel borocarbides [6,7] where $\kappa \sim 12$. However, this approach is less appropriate for low- $\kappa$ superconductors with large vortex cores such as $\mathrm{Nb}(\kappa \sim 0.774$ at $T_{c}$ [5]). Anisotropy of the superconducting energy gap [18] may also drive VL structural transitions in $d$-wave superconductors such as the heavy-fermion superconductor $\mathrm{CeCoIn}_{5}$ [8] and $\mathrm{YBa}_{2} \mathrm{Cu}_{3} \mathrm{O}_{7}$ [9]. Most models have been based upon the London or Ginzburg-Landau theories,

a)
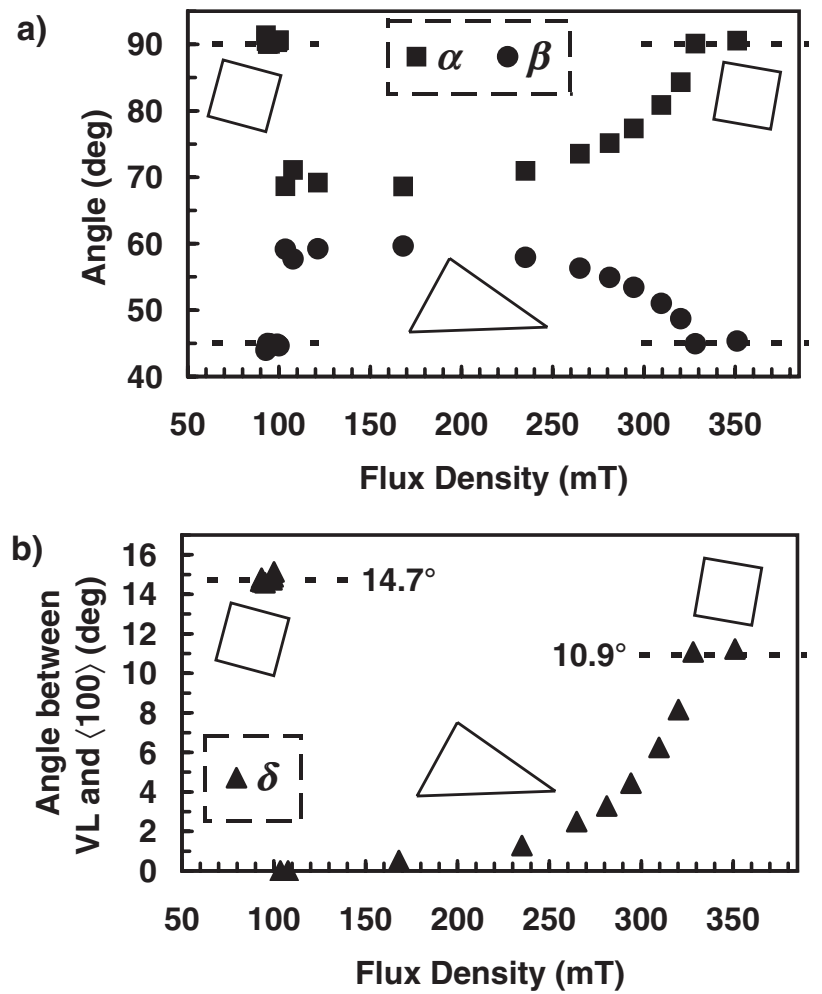

FIG. 5. The morphology of the vortex lattice as a function of flux density $B$ at $T=2 \mathrm{~K}$ in sample $\mathrm{Nb}-1$ : (a) the interior angles $\alpha, \beta$; (b) the orientation $\delta$ with respect to a $\langle 100\rangle$ crystal axis; these angles are described in Fig. 2. The shapes portray the structures observed in different field regions. 
which are only strictly valid in limiting cases - the London model for $H \ll H_{c 2}(T)$, the Ginzburg-Landau model for $T$ near $T_{c}$. Deep within the mixed state, and particularly at low $\kappa$, the greater generality of microscopic theory is required, such as the quasiclassical Eilenberger formulation [19] with gap anisotropy superimposed [20]. A recent study by Nakai et al. [21] adds to the Eilenberger equations anisotropies of both Fermi velocity and superconducting gap in the form of general $\cos 4 \phi$ terms, where $\phi$ is the polar angle about a fourfold crystal axis. For suitable values of the relative anisotropies, their effects can compete and lead to both a high-field square phase due to energy gap anisotropy and a low-field square phase due to Fermi surface anisotropy. The latter anisotropy is quite pronounced in $\mathrm{Nb}$ and has been well characterized by band-structure calculations [22] and experiments [23], and can explain the observed temperature dependence of the upper critical field, $H_{c 2}(T)$ [24]. The assignment of energy gaps to the different sheets of the Fermi surface is also important in calculating the gap anisotropy; however, despite much debate [25], these contributions have yet to be clearly determined for pure niobium.

We have seen that VL structures other than regular hexagonal can occur if there are anisotropic vortexinteraction terms (which possess the crystal symmetry in a conventional superconductor). If such terms depend on $\cos 4 \phi$, the VL structures with minimum free energy will orient along either the $\langle 100\rangle$ or $\langle 110\rangle$ axes. We point out that additional higher-order $\cos 8 \phi$ terms will allow minima which lie away from the directions of high crystal symmetry. A phenomenological theory of this kind could describe the $\sim 15^{\circ}$ and $\sim 10^{\circ}$ angles between nearest neighbors and $\langle 100\rangle$ crystal axes of the square VL phases. An explanation of the scalene-triangular structure would require a similar higher-order approach.

In conclusion, we have used small-angle neutron scattering to reexplore the morphology of the magnetic vortex lattice in pure niobium when the field is applied along a fourfold [001] direction. Our low-field results confirm earlier work, but we have discovered completely new aspects to the VL phase diagram at high fields and low temperatures. Both first- and second-order transitions between the various VL phases have been observed. It is clear that some degree of frustration is introduced into the system by the competition between the fourfold crystal symmetry and the tendency for vortex lines to form a triangular lattice. In the high-temperature "isosceles" phase, this frustration is resolved by forming a triangular VL which has mirror-plane symmetry about a $\langle 100\rangle$ axis, as would be expected from a Ginzburg-Landau theory with lowest-order nonlocal corrections. However, the low-temperature VL phases all break the crystal symmetry more comprehensively, and the high-field "scalene-triangular" VL phase has all nearest-neighbor directions at completely non-symmetrydetermined angles to the crystal axes. This spontaneous symmetry breaking contrasts with that of high- $T_{c}$ or bor- ocarbide superconductors in which the VL aligns with the crystal axes. At high and low fields, square VLs are observed, but again are not at symmetry-determined orientations. At the time of writing, only square VLs with nearestneighbor directions along $\langle 100\rangle$ or $\langle 110\rangle$ crystal axes have been anticipated in the literature $[9,18,20,21]$. However, a "conventional" superconductor such as niobium can still yield a cornucopia of surprising vortex structures.

This work is based on experiments performed at the Swiss spallation neutron source SINQ, Paul Scherrer Institut, Villigen, Switzerland, and at the Institut LaueLangevin, Grenoble, France. We acknowledge financial support from the Engineering and Physical Sciences Research Council of the U.K., H. Keller (University of Zürich), the Institut Laue-Langevin, the University of Birmingham, and from the European Commission under the 6th Framework Programme through the Key Action: Strengthening the European Research Area, Research Infrastructures, Contract No. RII3-CT-2003-505925.

[1] D. Cribier et al., Phys. Lett. 9, 106 (1964).

[2] A. A. Abrikosov, Sov. Phys. JETP 5, 1174 (1957).

[3] J. Schelten et al., J. Low Temp. Phys. 14, 213 (1974).

[4] D. K. Christen et al., Phys. Rev. B 21, 102 (1980).

[5] D. K. Christen and P. Thorel, Phys. Rev. Lett. 42, 191 (1979).

[6] D. McK. Paul et al., Phys. Rev. Lett. 80, 1517 (1998).

[7] V. G. Kogan et al., Phys. Rev. B 55, R8693 (1997).

[8] M. R. Eskildsen et al., Phys. Rev. Lett. 90, 187001 (2003).

[9] S. P. Brown et al., Phys. Rev. Lett. 92, 067004 (2004), and references therein.

[10] W. H. Kleiner et al., Phys. Rev. 133, A1226 (1964).

[11] B. Obst, Phys. Status Solidi B 45, 453 (1971).

[12] A. E. Jacobs et al., Phys. Rev. B 4, 3029 (1971).

[13] U. Essman, Phys. Lett. A 41, 477 (1972).

[14] D. K. Christen et al., J. Appl. Crystallogr. 11, 650 (1978).

[15] S. T. Sekula and R.H. Kernohan, Phys. Rev. B 5, 904 (1972); G. P. Van Der Mey, P. H. Kes, and D. De Klerk, Physica (Amsterdam) 95B+C, 369 (1978).

[16] V. G. Kogan et al., Phys. Rev. B 54, 12386 (1996); Phys. Rev. Lett. 79, 741 (1997).

[17] M. Yethiraj et al., Phys. Rev. B 72, 060504(R) (2005), and references therein.

[18] A. J. Berlinsky et al., Phys. Rev. Lett. 75, 2200 (1995); J. Shiraishi et al., Phys. Rev. B 59, 4497 (1999).

[19] G. Eilenberger, Z. Phys. 214, 195 (1968).

[20] M. Ichioka et al., Phys. Rev. B 59, 8902 (1999).

[21] N. Nakai et al., Phys. Rev. Lett. 89, 237004 (2002).

[22] L. F. Mattheiss, Phys. Rev. B 1, 373 (1970).

[23] M. H. Halloran, Phys. Rev. B 1, 366 (1970).

[24] W. H. Butler, Phys. Rev. Lett. 44, 1516 (1980).

[25] J. L. Bostock and M. L. A. MacVicar, in Anisotropy Effects in Superconductors, edited by H. Weber (Plenum, New York, 1977), p. 213; H.W. Weber et al., Phys. Rev. B 44, 7585 (1991); A. Hahn et al., Physica (Amsterdam) 296C, 103 (1998). 